NASA Technical Memorandum 101332 •

\title{
Modulated-Splitting-Ratio Fiber-Optic Temperature Sensor
}

Glenn Beheim

Lewis Research Center

Cleveland, Ohio

Donald J. Anthan and John R. Rys

Cleveland State University

Cleveland, Ohio

Klaus Fritsch

John Carroll University

Cleveland, Ohio

and

Walter A. Ruppe

Case Western Reserve University

Cleveland, Ohio

Prepared for

Fiber Optic and Laser Sensors VI

sponsored by the Society of Photo-Optical Instrumentation Engineers

Boston, Massachusetts, September 6-9, 1988 
Modulated-splitting-ratio fiber-optic temperature sensor

Glenn Beheim

National Aeronautics and Space Administration Lewis Research Center

Cleveland, Ohio 44135

Donald J. Anthan and John R. Rys

Department of Engineering Technology

Cleveland State University

Cleveland, Ohio 44115

Klaus Fritsch

Department of Physics

John Carroll University

Cleveland, ohio 44118

and

Walter R. Ruppe

Department of Electrical Engineering and Applied Physics

Case Western Reserve University

Cleveland, Ohio 441.06

\begin{abstract}
A fiber-optic temperature sensor is described that uses a small silicon beamsplitter whose splitting ratio varies as a function of temperature. A four-beam technique is used to measure the sensor's temperature-indicating splitting ratio. This referencing method provides a measurement that is largely independent of the transmission properties of the sensor's optical fiber link. A significant advantage of this sensor, relative to other fiber-optic sensors, is its high stability, which permits the fiber-optic components to be readily substituted, thereby simplifying the sensor's installation and maintenance.
\end{abstract}

\title{
1. INTRODUCTION
}

There is currently considerable interest in using fiber-optic sensors in aircraft control systems because of their light weight and immunity to electromagnetic interference (EMI). However, the high variability of fiber and connector losses makes it difficult to obtain the required accuracies. Simply remating a fiber connector or bending an optical cable can change the detected light level by several percent, thereby necessitating the use of some kind of light-level referencing scheme. Several different referencing techniques have been developed, of which, the spectrum-modulation or multiwavelength approach is most commonly used.l-1i This method uses a sensor that, as a function of the sensed variable, modifies the spectrum of the light that it transmits. This method requires that the Eiber link impose no further modification of the spectrum, however, in practice, appreciable errors can result due to the wavelength selectivity of the fiber-optic components. 12

A single-wavelength, four-beam referencing method that is analogous to the four-wire method of resistance measurement has been proposed by Giles, et al. I3 Giles' method uses an intensity-modulating sensor together with a number of highly stable optical splitters. We have improved on this method by using a beamsplitting sensor whose splitting ratio varies as a function of the sensed variable. Four separate light beams are used to obtain a measurement of the sensor's splitting ratio that is independent of the fiber link's transmissivity. Our approach has the advantage of not requiring highly stable splitters which are difficult to realize, particularly in the severe environment of the sensor. Several different pressure and displacement sensors have been described, and the performance of these laboratory prototypes has shown this method's effectiveness. $14-16$

In this report, we describe a modulated-splitting-ratio temperature sensor. A thin, uncoated silicon beamsplitter is interrogated by light having a wavelength of $830 \mathrm{~nm}$. As the temperature increases, so does the splitter's absorptivity,. thereby causing the transmitted light to decrease. Because the splitter is highly absorptive, nearly all the reflected light occurs due to the Fresnel reflection from the first surface. The reflectivity from this first surface is nearly temperature-insensitive; therefore, the sensor's splitting ratio (transmissivity divided by reflectivity) is the required monotonic function of temperature. 


\section{REFERENCING PRINCIPLE}

A generic modulated-splitting-ratio sensor is shown schematically in Fig. 1 . This sensor uses a beamsplitting transducer whose splitting ratio is a monotonic function of the measured variable. Two input fibers, $F-A$ and $F-B$, transmit the light from light-emitting diodes LED-A and LED-B to opposite sides of the beamsplitting transducer. Fused-fiber 2xl couplers direct the splitter outputs through the output fibers, F-U and F-V, to photodiodes PD-U and PD-V. The input and output fibers have connectors, $C$, at each end. The sensor is permanently attached to the couplers by fibers F-J and F-K. The sensor/coupler assembly uses fused fiber connections exçlusively. so as not to introduce any extraneous reflections.

The LEDS are operated sequentially, under the control of a microcomputer, so that the response of each photodiode to the light from each LED can be measured. The microcomputer uses two analog-to-digital converters (A/Ds) to digitize the amplified photodiode signals. The four digitized signals are designated $E_{\mathrm{NM}}$, where $E_{\mathrm{NM}}$ is the response of photodiode $N$ to the light from only LED $M$. These four signals are given by

$$
\begin{aligned}
& E_{U A}=s_{U} C_{U J} R_{J J} C_{J A} P_{A}, \\
& E_{U B}=s_{U} C_{U J}{ }^{T}{ }_{J K} C_{K B} P_{B}, \\
& E_{V A}=s_{V} C_{V K} T_{K J} C_{J A} P_{A}, \\
& E_{V B}=s_{V} C_{V K} R_{K K} C_{K B} P_{B} .
\end{aligned}
$$

In these equations, $C_{N M}$ denotes the transmission coefficient for light traveling from node $M$ to node $N$ of the fiber link, where the locations of the various nodes are shown in Fig. 1. The sensor transmission coefficients, TJK and $T_{K J}$, and reflection coefficients, $R_{J J}$ and $R_{K K}$, include the losses incurred upon coupling from the fiber denoted by the second subscript to the fiber denoted by the first subscript. The p variables are the output powers of the LEDs, and the $S$ variables are the sensitivities of the photodiode/amplifier/digitizer combinations, where, in both cases, the subscript identifies the LED or photodiode.

The microcomputer calculates a compensated sensor signal $Y$ from the four digitized photodiode signals, as follows:

$$
Y=\sqrt{\frac{E_{U B} E_{V A}}{E_{U A} E_{V B}}},
$$

Substituting from Eq. 1 yields

$$
Y=\sqrt{\frac{T_{J K} T_{K J}}{R_{J J^{R}} R_{K K}}} .
$$

The compensated signal $Y$ is equal to the sensor's splitting ratio and can therefore be used to infer the value of the measured variable. This instrument's output is highly stable; it is independent of the fiber, coupler and connector losses, as well as the coupler splitting ratios, LED output powers, photodiode sensitivities, and amplifier gains.

The preceding analysis has neglected several factors which may impair this referencing scheme's effectiveness. First, if the photometric system contains any nonlinearities or offsets, changes in the light levels can produce appreciable errors. Stray ambient light and light reflected from the connectors can also produce errors. A further source of error arises because this referencing scheme requires that the transmissivity for light traveling from node $M$ to node $N$ be the same, regardless of the path the 1 ight took before $M$ or after $N$. This requirement is never strictly satisfied by practical multimode fiber systems because of the mode-selectivity of the various components.

Besides the errors introduced by mode-selectivity, wavelength selectivity can produce essentially the same kind of effect by modifying the spectrum of the LED as the light travels through the fiber link. In this case, the transmissivity of a particular portion of the link depends on how the optical spectrum is modified by the other sections of the link through which the light travels. The sensor, in particular, must be wavelength independent, because, besides impairing the referencing scheme, a wavelength-selective sensor will have a splitting ratio that changes due to temperature-induced shifts in the LED wavelengths. 
References 14 to 16 have shown that relatively simple procedures can be used to reduce these referencing errors to a very low level. By using a four-fiber link, as shown in Fig. 1, the effects of connector reflections are greatly reduced when compared to the case of a two-fiber link (in the two-fiber system, the couplers are located locally and the optical signals are transmitted bidirectionally on connectorized fibers F-J and F-K). The effects of mode-selectivity can be made small by minimizing the separation within the sensor between the ends of fibers F-J and F-K. Finally, offsets can be compensated by the signal-processing algorithm. If these procedures are followed, stabilities permitting sensor accuracies of a percent or better are readily obtained.

\section{SENSOR DESCRIPTION}

Figure 2 shows a drawing of the modulated-splitting-ratio temperature sensor. The optical fibers have a core diameter of $100 \mu \mathrm{m}$ and a numerical aperture (N.A.) of 0.3 . A small piece of polycrystalline silicon serves as a temperature-sensitive beamsplitter. Fibers $\mathrm{F}-\mathrm{J}$ and $\mathrm{F}-\mathrm{K}$, which connect the sensor to the couplers, are $1-\mathrm{m}$ lengths of high-temperature optical fiber. This fiber has a polyamide buffer and a Teflon jacket and is rated for continuous use at $230{ }^{\circ} \mathrm{C}$. Two ferrules and a tube, all of stainless steel, are used to align the fiber ends. The sensor is assembled using an optical epoxy (Epo-Tek number 353 ND) that can be used continuously at 200 "C. First, fibers F-J and F-K are epoxied into the ferrules and their ends polished. A $100-\mu \mathrm{m}$ thick piece of polycrystalline silicon is then epoxied onto the end of F-J and the silicon is polished so that the light exiting F-J incurs a $15 \mathrm{~dB}$ loss in traversing the silicon. Using published values for silicon's refractive index 17 and absorption coefficient, 18 the splitter's thickness is estimated to be about $30 \mathrm{\mu m}$. The final step in the sensor's assembly is to epoxy the ferrules into the alignment sleeve and then cure the assembly at 200 "C for about 24 hr. Ihe assembled sensor has a length of about $25 \mathrm{~mm}$ and a diameter of $3 \mathrm{~mm}$.

For this proof-of-principle demonstration, the sensor's electronic system was built up using a number of laboratory instruments. Both the $830 \mathrm{~mm}$ wavelength LEDs and the silicon photodiodes are mounted on a common heat sink, whose temperature is $\mathrm{fixed}$ at $20 \pm 0.5{ }^{\circ} \mathrm{C}$ using a thermoelectric temperature-control unit. The photodiodes are operated in the photovoltaic mode in order to provide high linearity. The photodiode outputs are amplified using commercial instruments that have a gain of $10^{7} \mathrm{~V} / \mathrm{A}$ and a bandwidth of $10 \mathrm{kHz}$. The fiber-optic connectors are an optically contacting type (OFTI STC-type) that have low reflectivities when mated ( 0.4 percent). A personal computer, programmed using a compilable version of BASIC, and two IEEE-488 programmable voltmeters are used for signal acquisition and processing. The offsets are compensated in software using photodiode readings that are obtained with both LEDs off. The digital filters of the voltmeters are programmed so that the two offset voltages and the four sensor signals are measured in a total of $\sim 75$ ms This data acquistion rate is sufficient to compensate the relatively slow transmissivity changes that occur in our laboratory; in a high-vibration environment, a higher data rate may be needed.

\section{EXPERIMENTAL RESULTS}

A programable oven was used to test the sensor over the temperature range from 40 to $190^{\circ} \mathrm{C}$. The sensor's temperature. T, was measured using a type-K thermocouple together with a commercial ice-point reference and an IEEE-programmable voltmeter. Figure 3 shows measured values of $Y, E_{U A}$, and $E_{V A}$ as a function of $T$. A least squares fit to these data was used to express $T$ as a seventh-order polynomial function of $Y$. This polynomial expression was then used to convert subsequent $Y$-readings to a measured temperature, TM.

Figure 4 shows the measurement error, $\Delta T=T_{M}-T$, as a function of temperature for six different 40 to $190^{\circ} \mathrm{C}$ cycles, where only the data from the warming half of each cycle are plotted. During the first three cycles the optical link was left undisturbed. Next, the four input and output fibers were replaced and then left undisturbed for three more cycles. The maximum temperature error was $1.6^{\circ} \mathrm{C}$. The data from the cooling portions of these six cycles had approximately the same maximum error; no systematic hysteresis was observed. Figures 5 and 6 show the effects on $\Delta T$ of remating the fiber connectors for temperatures of 50 and $190^{\circ} \mathrm{C}$, respectively. Prior to each of 50 measurements of a fixed temperature, the four connectors attached to the coupler arms were demated and then remated. The RMS errors were 0.4 and $1.2{ }^{\circ} \mathrm{C}$, and the maximum errors were 0.7 and $1.8{ }^{\circ} \mathrm{C}$, at 50 and $190{ }^{\circ} \mathrm{C}$, respectively. For both experiments, the relative standard deviation of Eva was $\sim 5$ percent, indicating the fairly large variations in signal level that were successfully compensated.

Figure 7 shows the effects on $\Delta T$ of replacing the optical fibers for a temperature of $50{ }^{\circ} \mathrm{C}$. Prior to each of 30 temperature measurements, the four fibers used were selected at $r$ andom from a pool of eight fibers. Next, 30 more measurements were performed with each of the four fibers in the standard configuration replaced by two fibers joined together by a connector. Prior to each measurement, the positions of these eight fibers were randomly rearranged. For all 60 measurements, the RMS and maximum errors were 0.6 and $1.5{ }^{\circ} \mathrm{C}$, 
respectively. The signal level fluctuations during this experiment were quite large, the signals obtained using the eight-fiber link were, an"average of 60 percent lower than those obtained when only four Eibers were used.

The measurement error $\Delta \mathrm{T}$ was found to be linearly related to the LED/photodiode heatsink temperature, THS, for THS ranging from 10 to $30^{\circ} \mathrm{C}$. For a fixed sensor temperature, increasing $T_{H S}$ increases the LED wavelength, thereby increasing the splitting ratio due to the decrease in the absorption coefficient. The sensitivity of $\Delta T$ to $T_{H S}$ was measured for sensor temperatures of 50,100 and $200{ }^{\circ} \mathrm{C}$ and found to be $-0.56 \pm 0.02{ }^{\circ} \mathrm{C} /{ }^{\circ} \mathrm{C}$.

\section{CONCLUDING REMARKS}

The modulated-splitting-ratio sensor was found to be accurate within \pm 2 " $\mathrm{C}$ over the temperature range from 40 to $190^{\circ} \mathrm{C}$. The minimum temperature was determined solely by 1 imitations of our test apparatus; this sensor could probably be used to $-55{ }^{\circ} \mathrm{C}$ without any modification. The maximum temperature is limited by the properties of the epoxy used to bond the sensor. Significantly higher temperatures could be measured by using a different bonding method, such as a glass frit or: a glass-to-silicon anodic bond. An epoxy-less sensor could be expected to have the additional advantage of providing greater long-term stability. The long-term stability of our prototype sonsor needs further study: in particular, water absorption by the optical epoxy may prove to be a problem.

The four-fiber referencing system has been shown to be very effective; however, a twofiber system would be preferrable because it would be less expensive and easier to use. The two-fiber system requries that fibers $F-J$ and $F-K$ be connectorized and the optical signals be transmitted bidirectionally on them. For the referencing method to work effectively, these comnectors must have reflectivities of a few hundredths of a percent or less. These reflectively values are approximately an order of magnitude lower than the values we currently obtain using STC connectors. We are proceeding with experiments to determine if a different installation method can be used to reduce the reflectivity of these connectors.

\section{REFERENCES}

1. G. Beheim, K. Fritsch, and R.N. Poorman, "Fiber-Linked Interferometric Pressure Sensor," Rev. Sci. Instrum. 58(9), 1655-1659 (1987).

2. G. Beheim and K. Fritsch, "Spectrum-Modulating Fiber-optic Sensors for Aircraft Control Systems," NASA TM-88968 (1987).

3. G. Beheim, K. Fritsch, and D.J. Anthan, "Fiber Optic Temperature Sensor Using a Spectrum Modulating Semiconductor Etalon," in Fiber Optic and Laser Sensors V., R.P. DePaula and E. Udd, eds., Proc. SPIE 838, 238-246(1988).

4. J.C. Hartl, E.W. Saaski, and G.L. Mitchell, "Fiber Optic Temperature Sensor Using Spectral Modulation," in Fiber Optic and Laser Sensors V, R.P. DePaula and E. Udd. eds., Proc. SPIE 838, 257-263(1988).

5. P.W. Mossey, W.M. Shaffernocker, and A.R. Mulukutla, "17000 C Optical Temperature Sensor," NASA CR-175108 (1986).

6. K. Fritsch and G. Beheim, "Wavelength-Division Multiplexed Digital Optical Position Transducer," Opt. Lett. 11(1), 1-3 (1986).

7. G. Beheim, "Fibre-Optic Thermometer Using Semiconductor-Etalon Sensor," Electron. Lett. 22(5), 238-239 (1985).

8. M.M. Salour, G. Schoner, M. Kull, and J.H. Bechtel, "Semiconductor-platelet Fibre-Optic Temperature Sensor," Electron. Lett. 21(4), 135-136 (1985).

9. W.W. Morey, W.H. Glenn, and E. Snitzer, "Fiber Optic Temperature Sensor," in Proceedings of the 29 th International Instrumentation Symposium, Instrument Society of America, 261-274 (1983).

10. R.R. Dils, "High-Temperature Optical Fiber Thermometer," J. Appl. Phys. 54(3), $1198-1201(1983)$.

11. K. Kyuma, S. Tai, T. Sawada, and M. Nunoshita, "Fiber-optic Instrument for Temperature Measurement," IEEE J. Quantum Electron. QE-18(4), 676-679 (1982).

12. L.A. Johnson and S.C. Jensen, "Problems and Approaches for Remote Fiber Optic Absolute Sensors." in Fiber Optic and Laser Sensors III, E.M. Moore and O.G. Ramer, eds., Proc. SPIE $566,45-53(1985)$. 
13. I.P. Giles, S. McNeill, and B. Culshaw, "A Stable Remote Intensity Based Optical Fibre Sensor,"J. Phys. E: Sci. Instrum. 18(6), 502-504 (1985).

14. G. Beheim and D.J. Anthan, "Fiber-optic Photoelastic Pressure Sensor with Fiber-Loss-Compensation." Opt. Lett. 12(3), 220-222 (1987).

15. G. Beheim, "Loss-Compensation Technique for Fiber-Optic Sensors and Its Application to Displacement Measurements," Appl. Opt. 26(3), 452-455 (1987).

16. G. Beheim and D.J. Anthan, "Loss-Compensation of Intensity-Modulating Fiber-Optic Sensors," in Fiber Optic and Laser Sensors IV, R.P. DePaula and E. Udd, eds.. Proc. SPIE $718,259-265(1987)$.

17. Handbook of optical Constants of Solids, E.D. Palik, ed.. Academic Press, Orlando, FL, 1985, p. 565 .

18. W.C. Dash and R. Newman, "Intrinsic Optical Absorption in Single-Crystal Germanium and Silicon at $77^{\circ} \mathrm{K}$ and $300^{\circ} \mathrm{K}, "$ Phys. Rev. 99(4), 1151-1155 (1955).

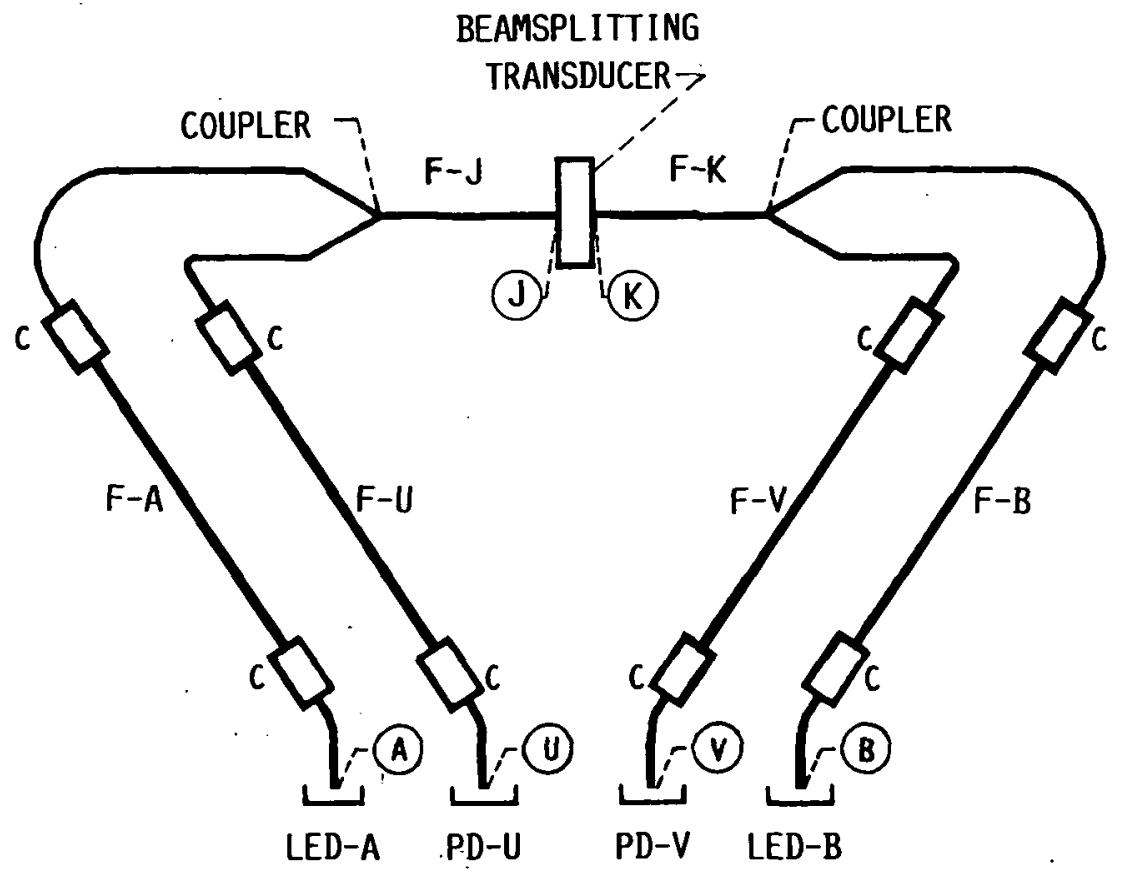

FIGURE 1. - MODULATED-SPLITTING-RATIO FIBER-OPTIC SENSOR. 


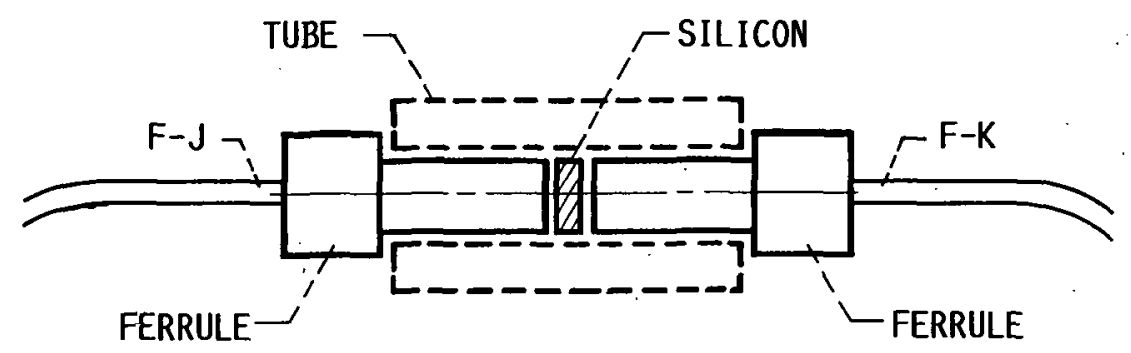

FIGURE 2. - MODULATED-SPLITTIING-RATIO TEMPERATURE SENSOR.

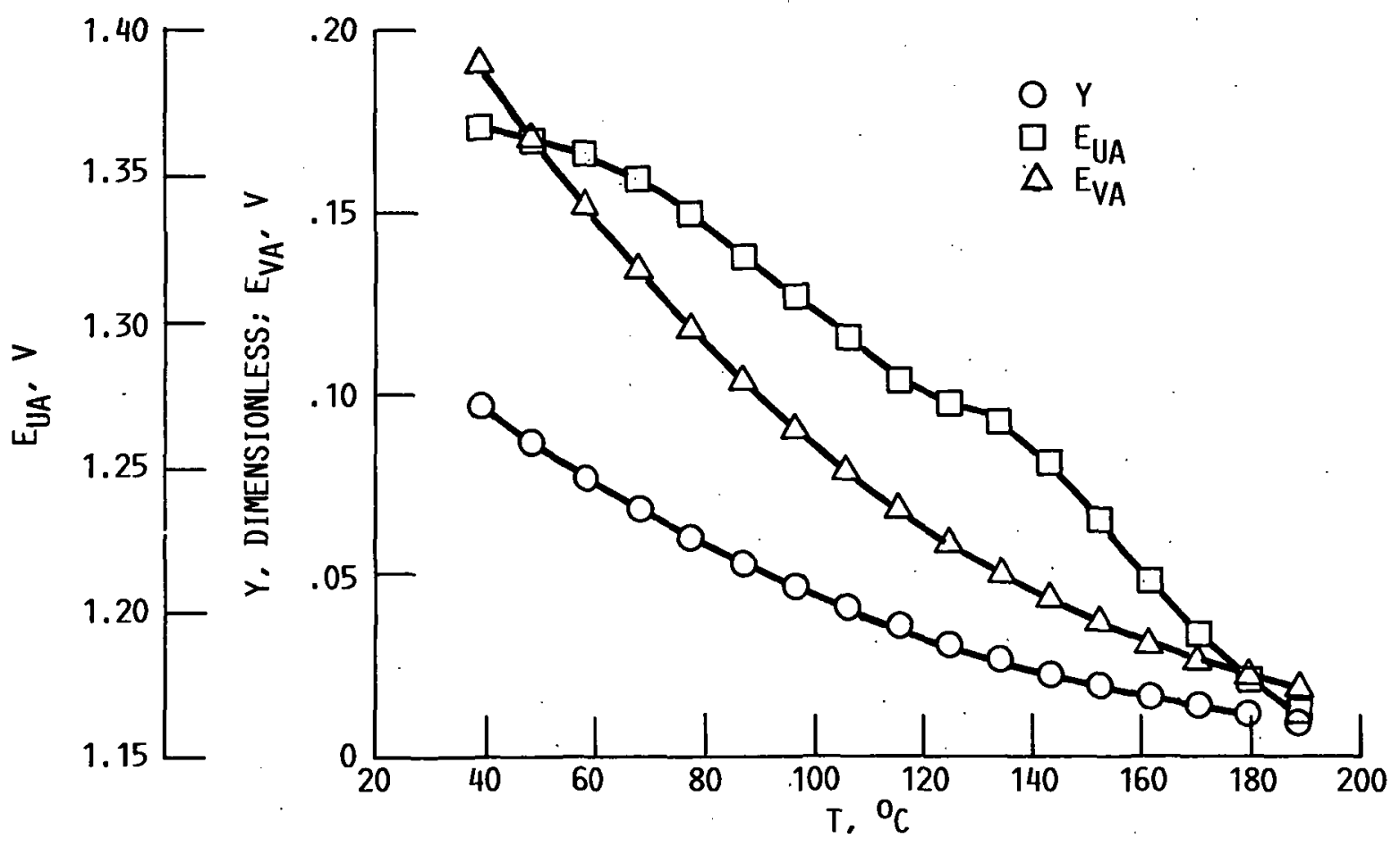

FIGURE 3. - SENSOR SIGNALS Y, EUA, AND EVA AS FUNCTIONS OF TEMPERATURE, $T$. 


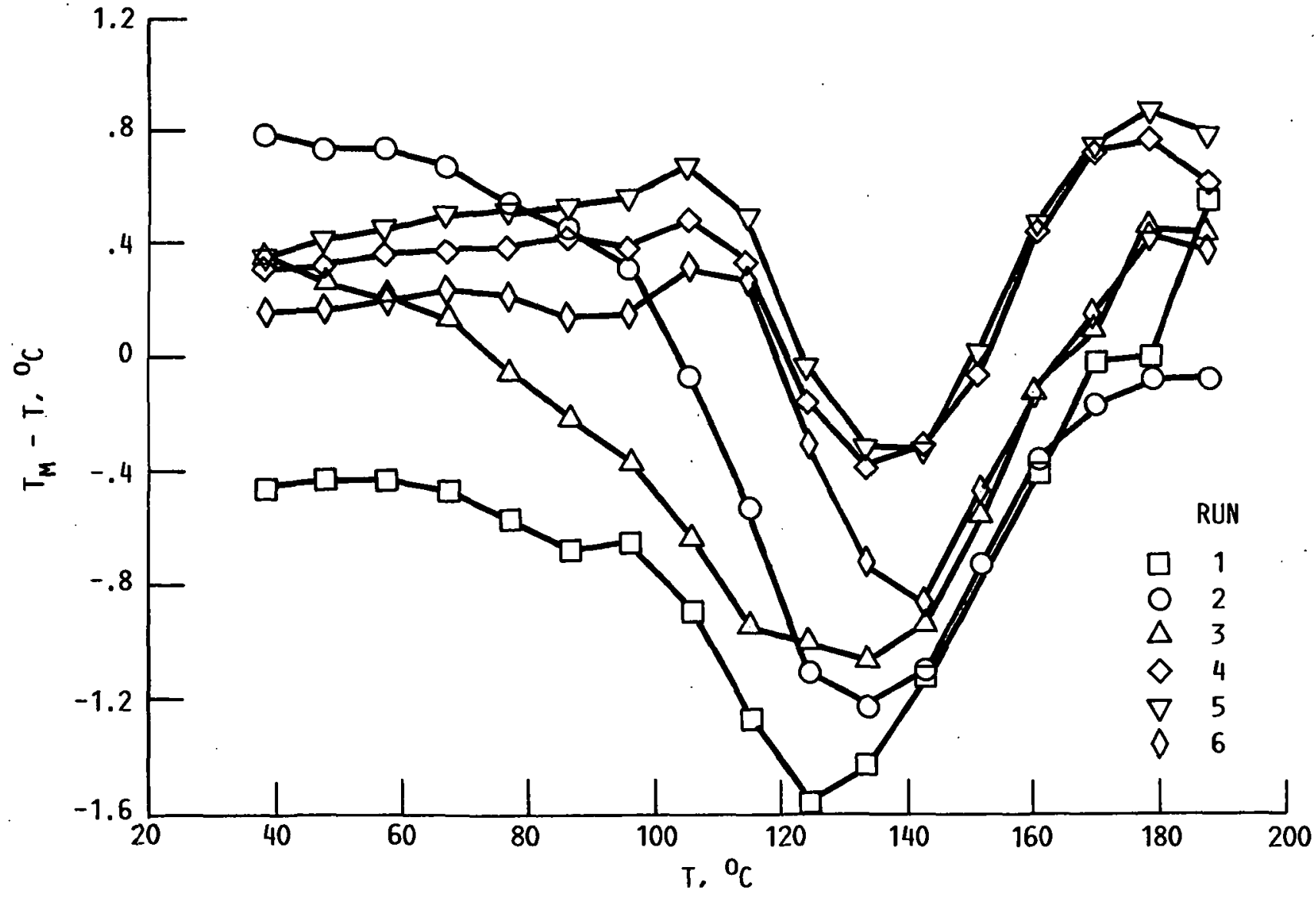

FIGURE 4. - TEMPERATURE ERROR, $\Delta T=T_{M}-T$, AS A FUNCTION OF $T$ FOR SIX $40-190{ }^{\circ} \mathrm{C}$ RUNS. 


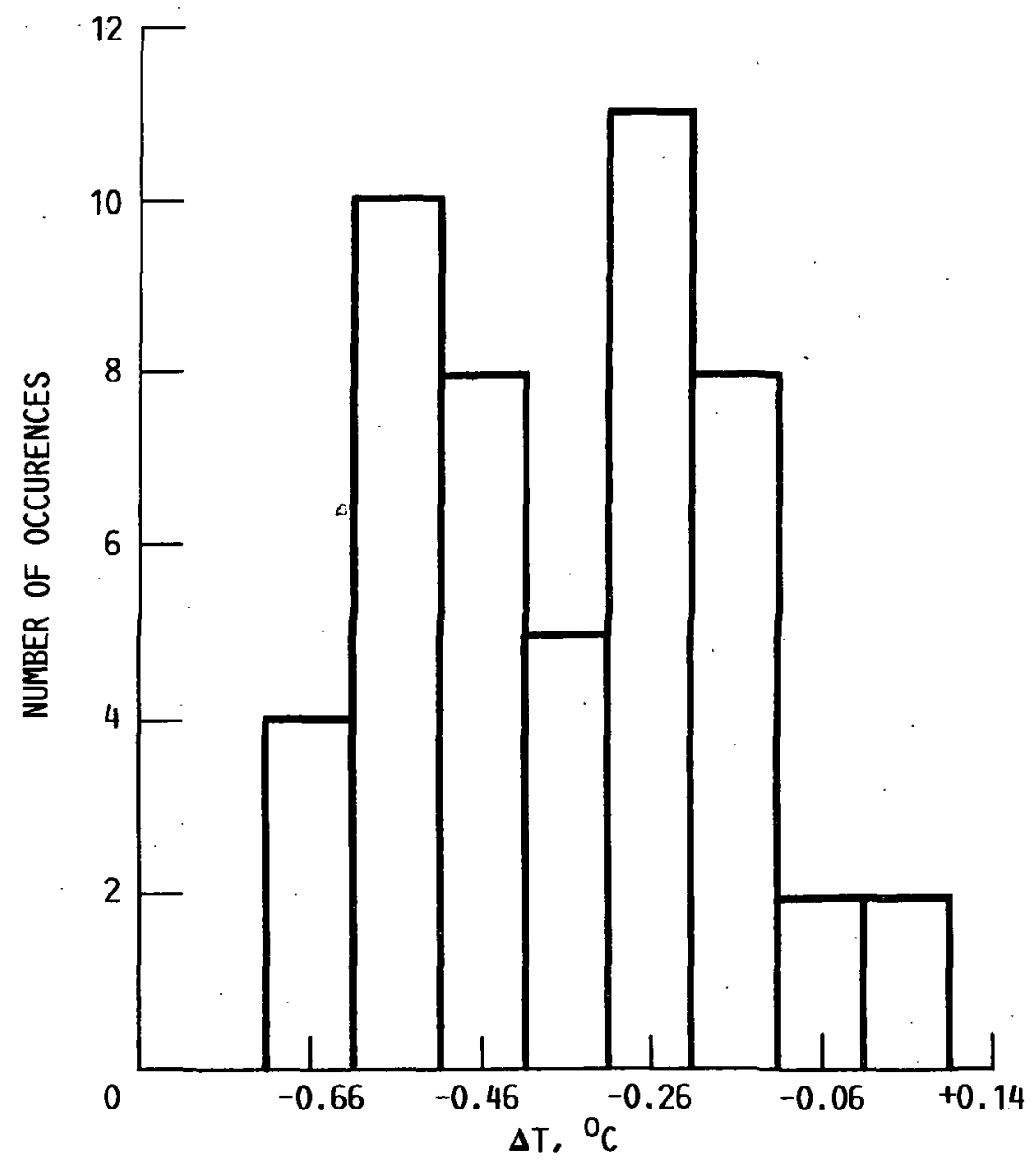

FIGURE 5. - EFFECT ON $\Delta T$ OF REMATING CONNECTORS AT. T $=50{ }^{\circ} \mathrm{C}$. 


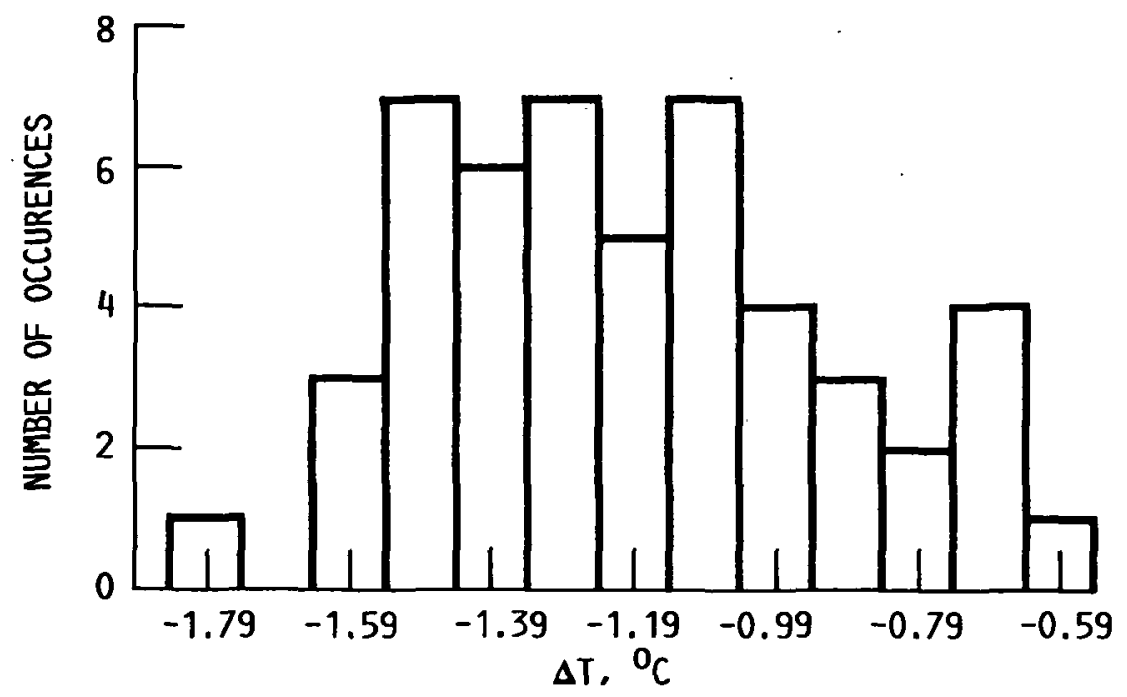

FIGURE 6. - EFFECT ON $\triangle T$ OF REMATING CONNECTORS AT $T=190{ }^{\circ} \mathrm{C}$.

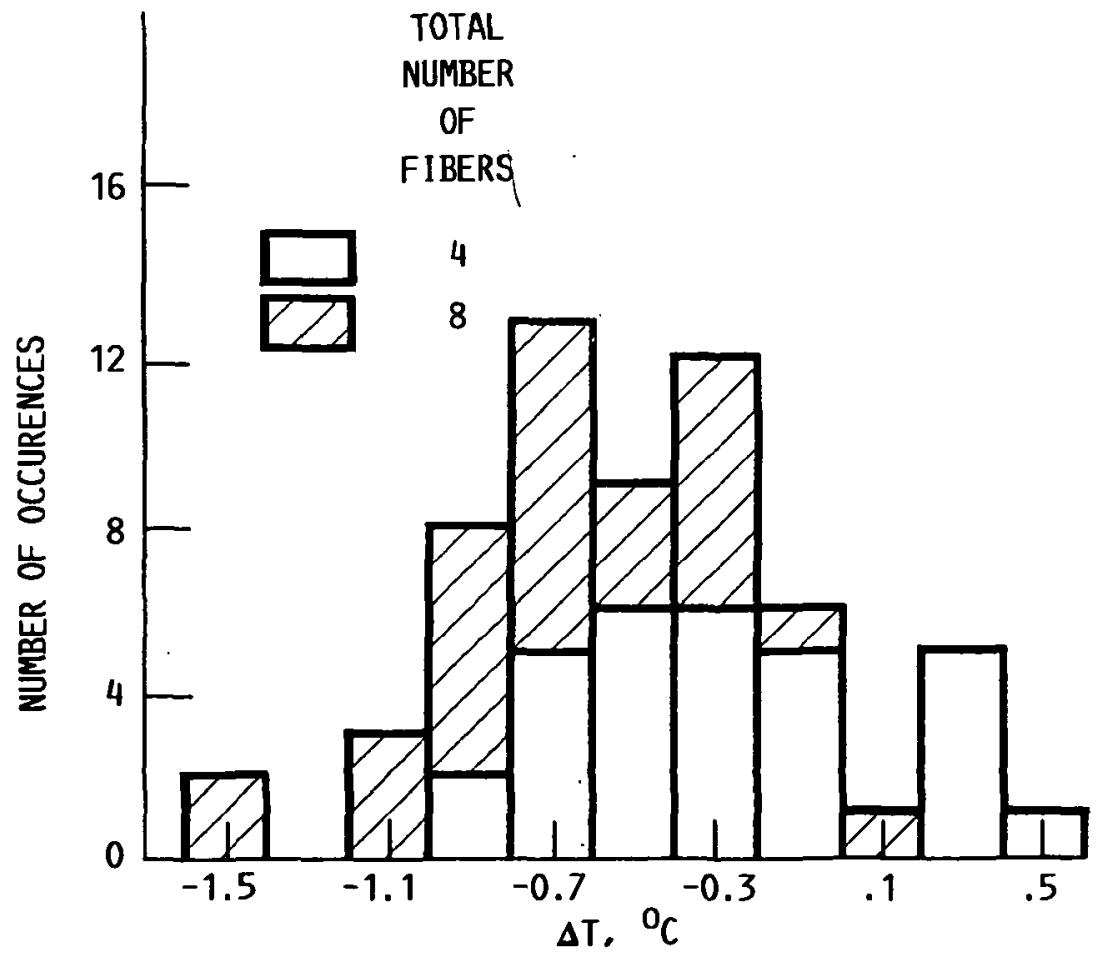

FIGURE 7. - EFFECT ON $\triangle T$ OF REPLACING FIBERS AT $\mathrm{T}=50^{\circ} \mathrm{C}$. 


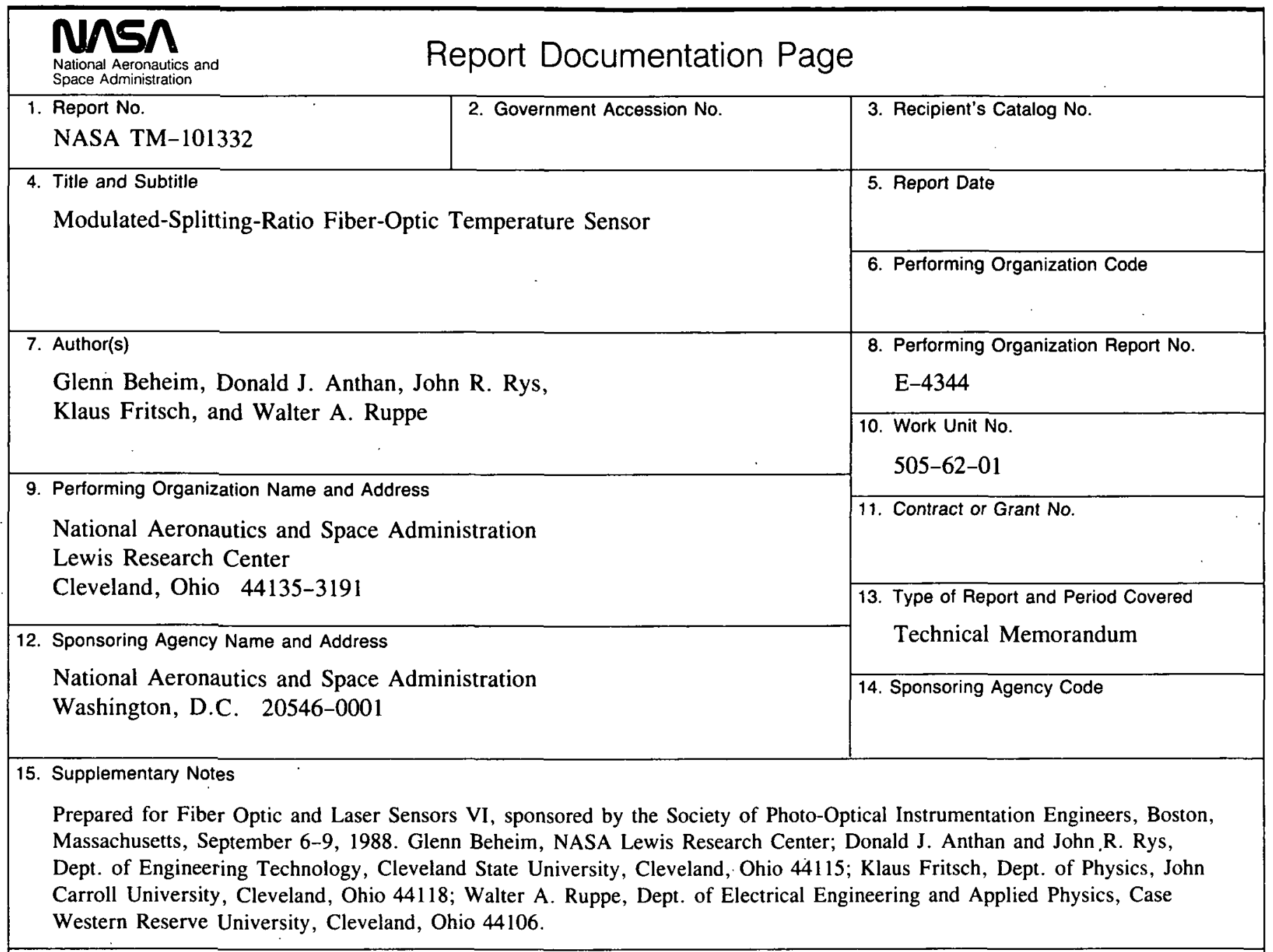

16. Abstract

A fiber-optic temperature sensor is described that uses a small silicon beamsplitter whose splitting ratio varies as a function of temperature. A four-beam technique is used to measure the sensor's temperature-indicating splitting ratio. This referencing method provides a measurement that is largely independent of the transmission properties of the sensor's optical fiber link. A significant advantage of this sensor, relative to other fiber-optic sensors, is its high stability, which permits the fiber-optic components to be readily substituted, thereby simplifying the sensor's installation and maintenance.

\begin{tabular}{|l|l|l|l|}
\hline $\begin{array}{l}\text { 17. Key Words (Suggested by Author(s)) } \\
\text { Fiber-optic sensors } \\
\text { Temperature sensors }\end{array}$ & $\begin{array}{r}\text { 18. Distribution Statement } \\
\text { Unclassified-Unlimited } \\
\text { Subject Category 06 }\end{array}$ \\
\hline $\begin{array}{c}\text { 19. Security Classif. (of this report) } \\
\text { Unclassified }\end{array}$ & $\begin{array}{c}\text { 20. Security Classif. (of this page) } \\
\text { Unclassified }\end{array}$ & $\begin{array}{r}\text { 21. No of pages } \\
10\end{array}$ & A02 Price* \\
\hline
\end{tabular}


National Aeronautics and Space Administration

Lewis Research Center Cleveland. Ohio 44135

Oncted Bushess

Pentity for Pitvato Uso \$500
SECOND CLASS MAIL

MODRESS CORRECTION REQUESTEO

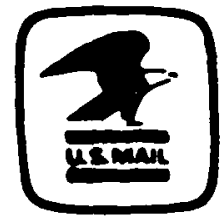

Postage and Fees Paid National Aeronautics and Space Administration NASA-451 